\title{
Corrigendum: The malaria parasite egress protease SUB1 is a calcium-dependent redox switch subtilisin
}

Chrislaine Withers-Martinez, Malcolm Strath, Fiona Hackett, Lesley F. Haire, Steven A. Howell, Philip A. Walker, Evangelos Christodoulou, Guy G. Dodson \& Michael J. Blackman

Nature Communications 5:3726 doi: 10.1038/ncomms4726 (2014); Published 2 May 2014; Updated 28 May 2014

The original version of this Article contained an error in the order in which the first and last name of the author Evangelos Christodoulou were displayed in the author list and as initials in the author contributions statement. This has now been corrected in both the PDF and HTML versions of the Article. 